\title{
A Hardware Efficient Reconfigurable Double-Balanced/Sub-Harmonic Down-Conversion CMOS Mixer for Cognitive Receiver Applications
}

\author{
Seongjin Bae, Dongmin Kim, Donggu Im \\ Division of Electronics Engineering, Chonbuk National University \\ Deokjin-gu, Jeonju-si, Republic of South Korea \\ tjdwls8126@ naver.com; aksd5736@naver.com; dgim@jbnu.ac.kr
}

\section{Extended Abstract}

Most recent and interesting evolution in the wireless communication area is the trend toward Internet of Things (IoT), which is a heterogeneous network of connected sensors and actuators attached to a wide variety of everyday objects. The multiple long-range, short-range, and personal area wireless networks and technologies are incorporated into the IoT platform. Many connectivity players predict that there will be an explosive growth within the next ten years and 50 billion IoT devices will be connected to the internet by 2020 [1]. In order to manage explosive demand on wireless data transmission traffic caused by countless IoT sensor nodes, the cognitive radio (CR) technology that recognizes the environment of surrounding spectrum and enables communication between conventional licensed devices and non-licensed devices without mutual interferences should be introduced to the IoT sensor nodes. By sensing unused "white spots" in the radio spectrum licensed to a primary device and dynamically and autonomously adjust its radio operating parameters while minimizing interference to the primary device, it improves the utilization efficiency of the scarce spectral resources [2].

One of the main challenges for the $\mathrm{CR}$ is the rapid and accurate spectrum detection in wideband for reliably detecting primary users before deciding on the frequency, channel, access, and modulation formats of transmission for the secondary users. Because the problem of primary user detection becomes much more complicated by multi-path fading and shadowing effects, the cognitive receiver should satisfy a high spurious free dynamic range (SFDR) of greater than $90 \mathrm{~dB}$ while having fast sensing time less than $200 \mathrm{msec}$. From the perspective of the receiver architectures, the fixed local oscillator (LO) based RF spectrum sensing receiver with the frequency channelization functionality, which is called channelizer [3], is considered one of the most competitive architectures because it can greatly reduce spectrum sensing time by removing an additional long settling time caused by the change of LO frequency. By down-converting a continuously occupied frequency band into the same intermediate frequency (IF) at the same time and sensing the multiple spectrums in parallel through the multiple baseband analog/digital chains, it can support fast spectrum sensing time with lower hardware complexity in comparison with that of the multiple LO based parallel RF spectrum sensing receiver. However, the complexity of the hardware is still high due to the repeated frequency conversion and filtering operation.

In this paper, the time-interleaved and channelized spectrum sensing receiver employing a reconfigurable doublebalanced and sub-harmonic down-conversion mixer is proposed to reduce the hardware complexity while maintaining fast sensing time. The proposed mixer is the passive type and supports three modes of $f_{I F}=\left|f_{R F}-2 f_{L O}\right|\left(2 f_{L O}\right.$ down-conversion mode), $f_{I F}=\left|f_{R F}-f_{L O}\right|\left(f_{L O}\right.$ down-conversion mode), and $f_{I F}=\left|f_{R F}\right|$ (by-pass mode) according to the digital control signal, where $f_{R F}, f_{L O}$, and $f_{I F}$ are the frequency of RF, LO, and IF. This operation enables a time-interleaved channelization with fast sensing time by switching the operation modes of the RF circuits instead of varying the LO frequency and as a result simplifies the hardware configuration of the conventional channelized spectrum sensing receiver. At $2 f_{L O}$ down-conversion mode, $f_{R F}$ of $1.9 \mathrm{GHz}$ and $f_{L O}$ of $0.9 \mathrm{GHz}$ are applied to the mixer in the simulation, and it shows a conversion loss of $4.1 \mathrm{~dB}$, a noise figure (NF) of 3.1 $\mathrm{dB}$, and an input-referred third-order intercept point (IIP3) of $+16 \mathrm{dBm}$. For $f_{L O}$ down-conversion mode, $f_{R F}$ of $1 \mathrm{GHz}$ and $f_{L O}$ of $0.9 \mathrm{GHz}$ are applied to the mixer, and it shows the conversion loss of $4.3 \mathrm{~dB}$, NF of $3.1 \mathrm{~dB}$, and IIP3 of $+18 \mathrm{dBm}$.

\section{Acknowledgements}

This research was supported by Basic Science Research Program through the National Research Foundation of Korea (NRF) funded by the Ministry of Education (NRF-2018R1A2B6008816). 


\section{References}

[1] Smooth Commerce's article, (2016, June 10). "50 billion IoT connected devices by 2020 will change business and life as we know it." [Online]. Available: http://smoothcommerce.tech/50-billion-iot-connected-devices-by-2020-willchange-business-and-life-as-we-know-it

[2] D. H. Mahrof, et al., "On the Effect of Spectral Location of Interferers on Linearity Requirements for Wideband Cognitive Radio Receivers," in the IEEE DySPAN, 2010.

[3] K. Jayaramam, "RF Frontend for Spectrum Analysis in Cognitive Radio," Ph.D. dissertation, Dept. Elect. Eng., Columbia Univ., USA, 2014. 